\title{
Suicide during pregnancy and in the first postnatal year
}

\author{
Louis Appleby
}

\begin{abstract}
Objectives-To calculate age adjusted mortality ratios for suicide by women in the first year after childbirth and during pregnancy, and to identify characteristics of postnatal suicide in childbearing women.
\end{abstract}

Design-Retrospective study based on population data for England and Wales from 1973 to 1984.

Subjects-Women aged 15-44 who committed suicide in the year after childbirth or during pregnancy.

Main outcome measures-Observed to expected mortality ratios for the total postnatal sample, for five year age groups, for unmarried mothers, and for suicide after stillbirth; observed to expected mortality ratios for all suicides during pregnancy and for five year age groups; the timing of suicides in relation to delivery; and the frequency of the various methods of suicide.

Results-The standardised mortality ratio for postnatal suicide was calculated to be $0 \cdot 17$-that is, the actual total was only one sixth of that expected. The low ratio was not found after stillbirth, which was associated with a rate six times that in all women after childbirth. The low ratio was less pronounced, but still present, in teenage mothers and in unmarried mothers. Women who committed suicide after childbirth most often did so in the first month, and there was a tendency to use violent methods. The standardised mortality ratio for suicide during pregnancy was calculated to be 0.05 of all pregnant women. Teenagers were at substantially increased risk.

Conclusions - Women in the first year after childbirth and during pregnancy have a low risk of suicide despite their high rate of psychiatric morbidity. Underreporting of maternal suicides is unlikely to explain the findings, though it may affect their magnitude. Motherhood seems to protect against suicide. Concern for dependants may be an important focus for suicide prevention in clinical practice.

\section{Introduction}

Increased rates of psychotic disorder and neurotic depression in women after childbirth have frequently been reported. ${ }^{1-4}$ A high rate of postnatal suicide might therefore be predicted. In pregnancy, although psychosis is rare, ${ }^{1}$ neurotic disorders are common ${ }^{2}$ and might similarly predict an increased suicide rate. This paper calculates the risk of suicide in the first postnatal year and during pregnancy by using population data for England and Wales from 1973 to 1984.

Previous attempts to study the relation between pregnancy and suicide in a locality have calculated the proportion of all women who commit suicide who are pregnant and have not adjusted for the effects of age. ${ }^{5}$ This study examines the effects of age and time trends on suicides in childbearing women. Some other characteristics of postnatal suicide are also examined.
Methods

Age standardised mortality ratios for suicides by women after childbirth and during pregnancy were calculated using the data sources and definitions listed below. In each case the data were broken down into five year age bands from 15 to 44 and into three year periods from 1973 to 1984.

Suicides in the general female population-Annual records of deaths by suicide and deaths in which cause - that is, accident or self harm-was undetermined were provided by the Office of Population Censuses and Surveys. It is conventional in statistical studies of suicide to regard the actual number of suicides as the sum of recorded suicides and recorded deaths of undetermined cause.

Population-Annual mid-year estimates of the female population of England and Wales stratified by age were provided by the Office of Population Censuses and Surveys.

Rates of suicide in women - The annual suicide rate per 100000 population was calculated for each age and period from the female suicides and population totals.

Births-Both livebirths and stillbirths were included in the postnatal calculation. The Office of Population Censuses and Surveys provided total numbers of births in England and Wales for each year stratified by age and marital state of the mothers. In calculating the rate during pregnancy the number of births was used rather than the number of conceptions so that the eventual mortality ratio would not artificially be lowered by the inclusion of pregnancies which ended in miscarriage or abortion. The expected total numbers of suicides, being based on annual estimates, were then multiplied by $34 / 52$ to adjust for the time during which a woman knows she is pregnant (assumed in this study to be 34 weeks).

Method of standardisation - The expected number of suicides in women after childbirth was calculated as the rate of suicide in women multiplied by the number of births. This calculation was carried out for each age group and time period.

Observed number of postnatal suicides-Details of all deaths in pregnancy and in the first postnatal year, classified by cause, are published every three years in the Report on Confidential Enquiries into Maternal Deaths in England and Wales. ${ }^{6}$ Cases are identified from death certificates completed by general practitioners and coroners and forwarded by district medical officers and the Office of Population Censuses and Surveys. Deaths are then recorded in three categories according to their relation to childbirth - namely, direct, indirect, and fortuitous. Suicides are either indirect or fortuitous, depending on the presumed influence of childbirth and pre-existing psychiatric illness. For each three year period the Department of Health provided additional information on maternal suicide, including age, marital state, obstetric outcome, timing of death in relation to delivery, method of suicide, coroner's verdict, and known psychiatric history. In this study maternal 
suicides included all of those listed by the Department of Health as suicide, except three cases in which the coroner's verdict was accidental death and one in which no inquest was held. In the remaining cases the coroner's verdict was either suicide, open, or misadventure, the last two corresponding to deaths of undetermined cause. Two suicides after legal abortion were excluded.

Mortality ratios-Ratios of observed to expected numbers of suicides were calculated for each three year period (all ages) and, separately, for each five year age band (all periods). An overall standardised mortality ratio was calculated after adding the individual age specific and period specific observed and expected numbers of suicides to produce total observed and expected numbers.

\section{Results}

SUICIDE IN FIRST POSTNATAL YEAR

Mortality ratios

Age standardised mortality ratios for each three year period were calculated. A test for heterogeneity ${ }^{7}$ showed the variation over time to be insignificant $\left(\chi^{2}=4 \cdot 7\right.$, $\mathrm{df}=3, \mathrm{p}=0 \cdot 2)$. An overall standardised mortality ratio was therefore calculated as the sum of observed suicides divided by the sum of expected suicides $=0 \cdot 17$ ( $95 \%$ confidence interval $0 \cdot 14$ to $0 \cdot 21$ ).

Table I shows the mortality ratio for each five year age band standardised for time. A test for heterogeneity showed no overall significant differences in the ratios $(p=0 \cdot 4)$, although the observed to expected ratio was highest in teenagers and generally declined with age. On testing for trend this decline failed to reach statistical significance $(p=0 \cdot 17)$.

TABLE I-Observed to expected ratios for postnatal suicide (1973-84). Results standardised for time period

\begin{tabular}{|c|c|c|c|c|c|c|}
\hline & \multicolumn{6}{|c|}{ Age (years) } \\
\hline & $15-19$ & $20-24$ & $25-29$ & $30-34$ & $35-39$ & $40-44$ \\
\hline Observed & 7 & 24 & 25 & 13 & 6 & 1 \\
\hline Expected & $19 \cdot 6$ & $127 \cdot 6$ & $165 \cdot 9$ & $92 \cdot 3$ & $35 \cdot 8$ & $8 \cdot 4$ \\
\hline Observed:expected & $0 \cdot 357$ & $0 \cdot 188$ & $0 \cdot 151$ & $0 \cdot 141$ & $0 \cdot 168$ & $0 \cdot 119$ \\
\hline
\end{tabular}

Test for heterogeneity: $\chi^{2}=5 \cdot 27, \mathrm{df}=5, \mathrm{p}=0 \cdot 4$.

TABLE II - Numbers of suicides in each four week period of year after childbirth (1973-84)

$\begin{array}{lrllllllllllllll}\text { Four week period after childbirth } & 1 & 2 & 3 & 4 & 5 & 6 & 7 & 8 & 9 & 10 & 11 & 12 & 13 & \text { Total }\end{array}$
No of suicides $\begin{array}{rrrrrrrrrrrrrr}1 & 2 & 3 & 4 & 5 & 6 & 7 & 8 & 9 & 10 & 11 & 12 & 13 & \text { Total } \\ 18 & 5 & 8 & 7 & 11 & 7 & 5 & 3 & 3 & 2 & 2 & 1 & 3 & 75 \star\end{array}$

${ }^{\star}$ In one case timing of suicide after childbirth was not recorded.

TABLE III-Methods of suicide among 76 women in year after childbirth

\begin{tabular}{lcccc}
\hline & Observed & Expected & $\begin{array}{c}\text { Observed: } \\
\text { expected }\end{array}$ & $\begin{array}{c}95 \% \text { Confidence } \\
\text { interval }{ }^{\star}\end{array}$ \\
\hline Overdose & 32 & 41.5 & 0.8 & 0.5 to 1.1 \\
Jumping from height & 11 & $4 \cdot 3$ & 2.5 & 1.3 to 4.6 \\
Self incineration & 9 & $2 \cdot 2$ & 4.0 & 1.8 to 7.8 \\
Drowning & 8 & $5 \cdot 7$ & 1.4 & 0.6 to 2.8 \\
Hanging & 7 & $7 \cdot 8$ & 1.0 & 0.4 to 2.0 \\
Jumping or lying in front of train & 7 & 3.3 & 2.1 & 0.8 to 4.4 \\
Self poisoning (not overdose) & 1 & 3.4 & 0.3 & 0.01 to 1.6 \\
\hline
\end{tabular}

*Confidence intervals from tables of means of Poisson distribution.

TABLE IV-Observed to expected ratios for suicide during pregnancy (1973-84)

\begin{tabular}{lcccccc}
\hline & \multicolumn{7}{c}{ Age (years) } \\
\cline { 2 - 7 } & $15-19$ & $20-24$ & $25-29$ & $30-34$ & $35-39$ & $40-44$ \\
\hline Observed & 5 & 3 & 4 & 2 & 0 & 0 \\
Expected & $17 \cdot 4$ & $88 \cdot 0$ & $101 \cdot 7$ & $51 \cdot 4$ & $18 \cdot 8$ & $4 \cdot 2$ \\
Observed:expected & $0 \cdot 280$ & $0 \cdot 034$ & $0 \cdot 039$ & $0 \cdot 039$ & 0 & 0 \\
\hline
\end{tabular}

Test for heterogeneity: $\chi^{\prime}=20 \cdot 35, \mathrm{df}=5, \mathrm{p}=0 \cdot 001$.

\section{Timing of postnatal suicide}

Table II shows the timing of the suicides in relation to delivery. Seventy five of the 76 suicides over the 12 years are included, the timing of suicide in one case having not been recorded. The data are presented as the number of suicides in each four week period of the first postnatal year. The mean monthly (that is, four weekly) figure was $5 \cdot 8$. The expected age standardised monthly mean was $34 \cdot 6$. The actual monthly totals varied widely with a large peak in the first month after delivery and a smaller possible peak at around five months. Even during the first month the number of suicides (18) was little more than half the rate expected from the general female population. After the second peak the numbers of suicides declined and remained at roughly one tenth of the expected number during the rest of the year.

\section{Other characteristics of postnatal suicide}

Stillbirths-Four of the women in the sample had had stillbirths (expected number in 76 births $=0.62$ ). The standardised mortality ratio for suicide after stillbirths was calculated to be 1.05 (95\% confidence interval 0.39 to 2.79 ) - that is, the rate was the same as in the general female population, therefore six times the rate in all women after childbirth.

Marital state-In 17 of the 76 suicides the women were unmarried (single, separated, or divorced). The standardised mortality ratio for suicide among unmarried mothers in the first year was calculated to be $0 \cdot 40$ ( $95 \%$ confidence interval 0.25 to $0 \cdot 64$ ). The rate of suicide in unmarried mothers was therefore less than half the rate in the general female population but more than twice the rate in all women after childbirth.

Psychiatric history-The Report on Confidential Enquiries into Maternal Deaths in England and Wales recorded a past or current psychiatric disorder in 48 of the 76 cases - that is, a proportion of $0 \cdot 63$. No further details were recorded, so that this figure was comparatively crude.

Method of suicide - Table III shows the methods of suicide compared with figures published by the Office of Population Censuses and Surveys on suicides and deaths of undetermined cause during 1974-84 (no publication in 1973). ${ }^{8}$ The expected figures were based on the rate of suicide (including deaths of undetermined cause) in the total female population aged 15-44 during 1974-84. Suicides by burning and by jumping in front of a train were listed individually from 1979; the expected figures for these methods were therefore based on the female population aged 15-44 from 1979 to 1984 . The observed to expected ratios suggested a high rate of dramatic methods of suicide, particularly self incineration, jumping from a height, and jumping in front of a train.

\section{SUICIDE DURING PREGNANCY}

Table IV shows the observed and expected numbers of suicides during pregnancy in the study period. A total of 14 suicides were reported in the 12 years compared with an expected $281 \cdot 5$, giving an overall observed to expected mortality ratio of $0.05(95 \%$ confidence interval 0.029 to 0.084 ) - that is, pregnant women had one twentieth of the expected rate of suicide. There was a significant age effect. The mortality ratio for teenage pregnant women was $0 \cdot 28$, so that, although at low risk compared with teenage non-pregnant women, this group carried a risk five times greater than pregnant women as a whole (test for heterogeneity: $\left.\chi^{2}=20 \cdot 35, \quad \mathrm{df}=5, \mathrm{p}=0.001\right)$. The numbers were too small to draw substantial conclusions about timing or method: Nevertheless, most suicides (eight of the 14) occurred in the second trimester. A psychiatric history was recorded in seven cases but in only one of the five cases in teenagers. 


\section{Discussion}

The principal finding of this study was a standardised mortality ratio for postnatal suicide of $0 \cdot 17$. At a time of high psychiatric morbidity the rate of suicide was roughly six times lower than expected in the matched female population. Possible explanations are considered below.

ERROR AND CHANCE

The accuracy of the low mortality ratio depends on the completeness of the numerator-that is, the maternal suicide sample. How comprehensive, then, was the method of data collection?

The Report on Confidential Enquiries into Maternal Deaths in England and Wales estimates that $99 \%$ of all direct puerperal deaths (that is, deaths in the first six weeks) and a slightly lower percentage of indirect puerperal deaths are included in its sample. ${ }^{6}$ Data on late deaths (after six weeks) and fortuitous deaths are less complete but the exact extent of underreporting is unknown. So it is possible that the low mortality ratio, or certainly the low number of maternal suicides after seven months, might have been the result of incomplete sampling. Several points, however, militate against this. Only if $83 \%$ of maternal suicides had been missed would the low ratio be cancelled out, and even greater underreporting would be needed to give a suicide rate consistent with the high rate of postnatal morbidity. Secondly, during the first postnatal month, when major morbidity is at a peak and suicide reporting relatively complete, the observed number of suicides was still well below the expected number (18 as opposed to $34 \cdot 6$ ). Thirdly, the pattern of maternal suicide, including the fall in cases after seven months, might satisfactorily be explained by the known pattern of maternal morbidity. Fourthly, most equivocal cases supplied by the Report on Confidential Enquiries into Maternal Deaths in England and Wales were included in the calculation.

An accurate check on the completeness of the data on suicides would require extensive cross matching of birth and death registers and would itself be a substantial study with its own methodological flaws. A simpler method is to select a fatal condition that could not influence or be influenced by fertility or childbirth (this excludes vascular disease, accidents, and tumours) and carry out an analogous calculation of the expected number of deaths in the childbearing population. This was done for meningitis during 1976-81. The expected number of deaths was $2 \cdot 4$; the observed number was 2 . Such small figures can be only minor evidence, but it may be significant that there was no large discrepancy, as there was with suicide.

The 95\% confidence interval of the standardised mortality ratio $(0.14$ to 0.21$)$ makes it unlikely that such a low ratio would be a statistical artefact.

\section{LOW PSYCHIATRIC MORBIDITY}

Recently the reported excess of neurotic depression in the first few months after childbirth ${ }^{2+}$ has been questioned, ${ }^{90}$ but there is no evidence of any postnatal decline in the rate of depression. Furthermore, the rate of psychotic illnesses increases dramatically in the puerperium. ${ }^{1}$ The low standardised mortality ratio is therefore unlikely to reflect a low rate of morbidity.

\section{HEALTHY SAMPLE}

Except with respect to marital state there was no information on whether the childbearing population in 1973-84 was low in risk factors for depression or suicide-for example, a psychiatric history. As for marital state itself, the rate of suicide in unmarried mothers alone was well below the rate in the general female population (standardised mortality ratio $0 \cdot 4$ ).

The remaining two explanations accept that women after childbirth are protected against the expected risk of suicide despite high psychiatric morbidity.

\section{FAMILY AND PROFESSIONAL SUPPORT}

The first postnatal year is a time of close contact with health professionals and family or friends. It may be that the risk of suicide is reduced by early detection and close support. Table II, however, shows the highest risk of suicide to occur in the first postnatal month, probably the time of greatest contact with professionals and support from family members.

PROTECTIVE EFFECT OF MOTHERHOOD

The remaining explanation is that being a mother is in itself protective against suicide. An American study of reasons for living ${ }^{11}$ found that in a sample of psychiatric inpatients those who had had suicidal thoughts and those who had actually carried out a parasuicidal act were distinguished by "child-related concerns." Specifically, these were $(a)$ that suicide would harm the children, $(b)$ that it would not be fair to leave the children to the care of someone else, and $(c)$ a desire to watch the children grow up. Such a result is in keeping with the large volume of research on cognitive aspects of depression. ${ }^{2}$

Could the same concerns about the children exert a protective effect on women in the first year after childbirth? There seems to be supportive evidence in this sample. Firstly, the absence of a low mortality ratio after stillbirth supports the presence of young children as the crucial protective influence. Each of the four stillbirths was a first pregnancy, so no older children could compensate. Secondly, the continuing low risk of suicide at the end of the first postnatal year (table II) would be expected if concerns over young children were indeed protective. Thirdly, the data suggest (but do not prove) that many of the suicides occurred during psychosis-for example, the peak during the first month coincided with the peak of onset of psychosis, ${ }^{113}$ and the methods used were more dramatic than expected. It may be during acute psychosis that rational child concerns are most disrupted.

Similarly, suicide during pregnancy does not mirror either the rate or the timing of neurotic disorders. It is a rare event throughout pregnancy, including during the first trimester, when non-psychotic morbidity is common. ${ }^{2}$ The very low mortality ratio during pregnancy, when child related concerns should be at their most direct, supports the conclusion that such concerns are a powerful influence on risk of suicide. Underreporting of suicides to the confidential enquiries into maternal deaths is thought to be fairly insignificant in pregnancy.

Although the prevalence of suicide in the general population increases with age, within the population of pregnant women teenagers seem to be at considerably greater risk. Most of the suicides in teenagers in this study were in women who had no recorded past or recent psychiatric history; this differs from the setting in older women, which suggests that any link between psychosis and suicide in this population may not apply to teenage women. The explanation may be that as pregnancy seems to be more often unwanted among teenagers (the abortion rate is higher ${ }^{14}$ ) the negative impact bf becoming pregnant is likely to be greater and the protective effect of motherhood reduced. If this is so, teenagers who are unhappy about being pregnant represent a high risk group within a low risk population.

\section{Conclusions}

Despite theoretical justification, the low risk of suicide in childbearing women should be confirmed 
with further studies. One method already mentioned is a cross matching of national birth and death registers, which should become easier once a computer link exists to allow a detailed comparison of information about subjects listed. Another approach is to use regional obstetric registers or national census data to relate death by suicide to childbirth or child age. These methods are currently being pursued.

If the low risk is confirmed, then more exploration of the possible reasons would be valuable. If there is a large protective effect of motherhood based on concern for the welfare of dependants such concern would be an important focus for suicide prevention work, both with postnatal patients and with others at risk.

This work was made possible by the cooperation of $\mathrm{Dr}$ Elizabeth Cloake, of the Department of Health, and the staff of the Office of Population Censuses and Surveys. Dr Glyn Lewis, of the Institute of Psychiatry, provided statistical advice.

1 Kendell RE, Chalmers JC, Platz C. Epidemiology of puerperal psychoses. Brf Psychiatry 1987;150:662-73.

2 Kumar R, Robson KM. A prospective study of emotional disorders in childbearing women. $B r \mathcal{F}$ Psychiatry 1984;144:35-47.
3 Paffenbarger RS. Epidemiological aspects of parapartum mental illness. British fournal of Preventive and Social Medicine 1964;18:189-95.

+ Watson JP, Elliott SA, Rugg AJ, Brough DI. Psychiatric disorder in pregnancy and the first postnatal year. Br $\mathcal{F} P$ sychiatry 1984;144:453-62.

Kleiner GJ, Greston WM, eds. Suicide in pregnancy. Littleton, Massachusetts: John Wright, 1984

6 Department of Health and Social Security. Report on confidential enquiries into maternal deuths in England and Wales. London: HMSO, 1979, 1982, 1986, 1990. (Triennial publication.

7 Breslow NE, Day NE. Statistical methods in cancer research. Vol 2. The design and analysis of cohort studies. Lyons: International Agency for Research on and analysts of

8 Office of Population Censuses and Surveys. Mortality statistics: cause. London: HMSO, 1974-84. (Annual publication.)

9 O'Hara MW, Zckoski EM. Postpartum depression: a comprehensive review. In: Kumar R, Brockington IF, eds. Motherhood and mental illness. 2. Cause In: Kumar R, Brockington IF, eds. Motherhood
and consequences. London: Wright, 1988:17-63.

10 Cooper PJ, Campbell EA, Day A, Kennerley H, Bond A. Non-psychotic psychiatric disorder after childbirth; a prospective study of prevalence, incidence, course and nature. $B r f$ Psychiatry 1988;152:799-806.

11 Linehan MM, Goodstein JL, Nielsen SL, Chiles JA. Reasons for staying alive when you are thinking of killing yourself: the reasons for the living inventory. F Consult Clin Psychol 1983;51:276-86.

12 Beck AT, Rush J, Shaw BF, Emery G. Cognitive therapy of depression. New York: Guildford Press, 1979.

13 Brockington I, Cox-Roper A. The nosology of puerperal mental illness. In Kumar R, Brockington IF, eds. Motherhood and mental illness. 2. Causes and consequences. London: Wright, 1988:1-16.

14 Office of Population Censuses and Surveys. Birth statistics. London: HMSO, 1973-84. (Annual publication.)

(Accepted 7 September 1990)
Vale of Leven Hospital, Alexandria,

Dunbartonshire G83 0UA

N A Miller, MRCP, clinical research assistant

H A Carmichael, FRCP, consultant physician

F C Hall, BSC, biochemist

Helensburgh,

Dunbartonshire

B D Calder, MRCGP, general

practitioner

University of Glasgow,

Glasgow G12 8QQ

$\mathrm{PO}$ Behan, MD, professor in

neurology

Regional Virus Laboratory, Ruchill Hospital,

Glasgow

E J Bell, MRCPATH, top grade virologist

R A McCartney, FIMLs, chief medical laboratory scientific officer

Correspondence to:

Dr Carmichael.

BMF 1991;302:140-3

\title{
Antibody to coxsackie B virus in diagnosing postviral fatigue syndrome
}

\author{
N A Miller, H A Carmichael, B D Calder, P O Behan, E J Bell, R A McCartney, F C Hall
}

\section{Abstract}

Objective-To study the association between coxsackie $B$ virus infection and the postviral fatigue syndrome and to assess the immunological abnormalities associated with the syndrome.

Design-Case-control study of patients with the postviral fatigue syndrome referred by local general practitioners over one year.

Setting-General practitioner referrals in Dunbartonshire, Scotland.

Patients - 254 Patients referred with the postviral fatigue syndrome (exhaustion, myalgia, and other symptoms referable to postviral fatigue syndrome of fairly recent onset-that is, several months) and age and sex matched controls obtained from same general practitioner; 11 patients were rejected because of wrong diagnoses, resolution of symptoms, and refusal to participate, leaving 243 patients and matched controls.

Main outcome measures-Detailed questionnaire (patients and controls) and clinical examination (patients) and blind analysis of blood sample at entry and after six months for determination of coxsackie $B$ virus IgM and IgG antibodies and other variables (including lymphocyte protein synthesis, lymphocyte subsets, and immune complexes).

Results-Percentage positive rates for coxsackie $B$ virus IgM at entry were $24.4 \%$ for patients and $22.6 \%$ for controls and for coxsackie $B$ virus IgG $56.2 \%$ and $55.3 \%$ respectively; there were no significant differences between different categories of patients according to clinical likelihood of the syndrome nor any predictive value in a fourfold rise or fall in the coxsackie $B$ virus IgG titre in patients between entry and review at six months. The rates of positive antibody test results in patients and controls showed a strong seasonal variation. Of the numerous immunological tests performed, only a few detected significant abnormalities; in particular the mean value for immune complex concentration was much higher in $\mathbf{3 5}$ patients and 35 controls compared with the normal range and mean value for total IgM was also raised in 227 patients and 35 controls compared with the normal range.

Conclusions-Serological tests available for detecting coxsackie $B$ virus antibodies do not help diagnose the postviral fatigue syndrome. Percentage positive rates of the antibodies in patients simply reflect the background in the population as probably do the raised concentrations of total IgM and immune complexes.

\section{Introduction}

Since 1934 numerous outbreaks of a curious ill defined illness showing similarities in presentation, clinical picture, and outcome have been reported throughout the world under various synonyms, the most recent being the postviral fatigue syndrome and the chronic fatigue syndrome. ${ }^{1-4}$ Outbreaks of the illness have been associated with increased antibody titres to coxsackie B virus. ${ }^{5-9}$ One of us (HC) noted that between 1980 and 1984 the annual referral rate of medical outpatients in Dumbarton district thought to have the postviral fatigue syndrome more than quadrupled and seemed to be associated with high positive rates of coxsackie B IgG antibodies (about $40 \%$ each year) compared with the previously reported background rate of positive tests. ${ }^{10} \mathrm{With}$ the advent of a coxsackie $B$ virus $\operatorname{IgM}$ antibody test the present prospective trial was set up to assess any association between the postvirual fatigue syndrome and coxsackie $B$ virus infection and to examine immunological abnormalities previously described in this syndrome. ${ }^{8}$

\section{Materials and methods}

Patients and controls

During one year starting on 1 May 1985 local general practitioners were asked to refer patients with 